\title{
Use of Antiepileptic Drugs in Aneurysmal Subarachnoid Hemorrhage
}

\author{
Adam A. Dmytriw (D), Georgios A. Maragkos, Jeffrey Zuccato, Jeffrey M. Singh, \\ M. Elizabeth Wilcox, Sacha Schweikert
}

\begin{abstract}
Introduction: Controversy exists in antiepileptic drug (AED) prophylaxis prescribing in patients with aneurysmal subarachnoid hemorrhage (SAH). We undertook the Use of Antiepileptic Drugs in Aneurysmal Subarachnoid Hemorrhage (ALIBI) study to identify factors associated with prescribing practices. Methods: A retrospective chart review of all consecutive patients requiring Level 1 care with aneurysmal SAH admitted between 2012 and 2014 to the intensive care unit at Toronto Western Hospital, Ontario, Canada, was conducted. Data were collected on clinical and imaging characteristics. Primary and secondary outcomes were AED prophylaxis and clinical seizure activity during hospitalization. Data were compared using chi-square or Mann-Whitney U-tests. Those variables found to be significant, or trending toward significance, on univariate analysis were fitted to multivariate regression. Results: Sixty-eight patients were included. Mean age was $62 \pm 12.2$, and $42.6 \%$ of patients were male. Of these, 21 patients (30.9\%) received AED prophylactically, while 18 (26.5\%) had reported seizures at some point during hospitalization. Female gender and presence of midline shift (MLS) were significantly associated or approached significance with AED prophylaxis in univariate analysis $(\mathrm{p}=0.036$ and $\mathrm{p}=0.062$, respectively). In multivariate analysis, only MLS was an independent predictor (odds ratio 5.09, $\mathrm{p}=0.04$ ). Conclusion: The presence of MLS was an independent predictor of seizure activity in patients with aneurysmal SAH. AED prophylaxis prescribing patterns seemed arbitrary and was not informed by identifiable clinical factors or true risk factors for seizure. A current lack of evidence guiding AED prescribing practice highlights the need for larger studies in this patient population.
\end{abstract}

RÉSUMÉ: Utiliser des médicaments antiépileptiques dans des cas d'hémorragie sous-arachnoïdienne anévrismale. Introduction: Dans les cas de patients victimes d'hémorragie sous-arachnoïdienne anévrismale, il faut savoir qu'un traitement prophylactique au moyen de médicaments antiépileptiques demeure controversé. Dans cette étude, nous avons donc entrepris d'identifier les facteurs associés aux pratiques de prescription de ces médicaments lorsque survient ce type d'hémorragie. Méthodes: Pour ce faire, nous avons passé en revue les dossiers de tous les patients vus consécutivement et ayant nécessité, après avoir été admis entre 2012 et 2014 à l'unité des soins intensifs du Toronto Western Hospital (Ontario, Canada), des soins de niveau 1 à la suite d'une hémorragie sous-arachnoïdienne anévrismale. Nous avons collecté nos données en nous basant sur des aspects cliniques et sur d'autres aspects liés à des examens d'IRM. Notre principal résultat mesuré a été l'efficacité d'un traitement prophylactique au moyen de médicaments antiépileptiques; dans un deuxième temps, nous avons aussi cherché à mesurer l'activité convulsive clinique en cours d'hospitalisation. Nous avons ensuite comparé nos données en utilisant les tests du X2 ou de U Mann-Whitney. Les variables apparues significatives ou tendant à être significatives dans le cadre d'une analyse univariée ont été ajustées pour une régression multivariée. Résultats: 68 patients ont été inclus dans cette étude. Leur âge moyen était de $62 \pm 12,2$; $42,6 \%$ d'entre eux étaient des hommes. De ce nombre, 21 patients $(30,9 \%)$ ont alors suivi un traitement prophylactique au moyen de médicaments antiépileptiques tandis que 18 (26,5\%) ont fait état de crises convulsives à un moment ou un autre de leur séjour à l'hôpital. Dans le cadre d'une analyse univariée, le fait d'être une femme et la présence d'une déviation de la ligne médiane (midline shift) ont été par ailleurs nettement associés ou en grande partie associés à un traitement prophylactique au moyen de médicaments antiépileptiques (respectivement $\mathrm{p}=0,036$ et $\mathrm{p}=0,062$ ). Dans le cadre d'une analyse multivariée, seule la déviation de la ligne médiane s'est avérée un facteur prédicteur indépendant (rapport des cotes de 5,09; $\mathrm{P}=0,04)$. Conclusion: La présence de déviation de la ligne médiane constitue un facteur prédictif indépendant d'activité convulsive chez des patients victimes d'hémorragie sous-arachnoïdienne anévrismale. De plus, les tendances en matière de prescription de médicaments antiépileptiques apparaissent arbitraires et ne reposent pas sur des facteurs cliniques identifiables ou sur de véritables facteurs de risque liés aux convulsions. Le manque actuel de preuves pouvant orienter les pratiques de prescription de ces médicaments met en lumière la nécessité d'effectuer de plus amples études au sein de cette population de patients.

Keywords: Subarachnoid hemorrhage, Antiepileptic drugs, Seizure prophylaxis, Neurocritical care doi:10.1017/cjn.2019.54

Can J Neurol Sci. 2019; 46: 423-429

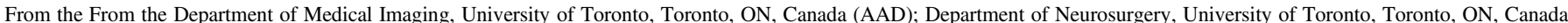

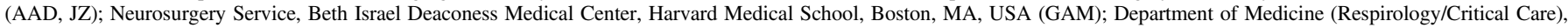

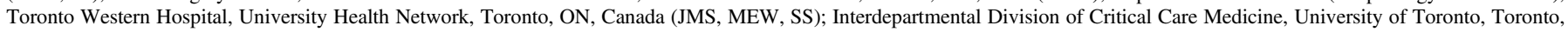
ON, Canada (JMS, MEW)

Received October 22, 2018. Final Revisions Submitted March 26, 2019. Date of Acceptance April 10, 2019.

Correspondence to: Adam A. Dmytriw, Department of Medical Imaging, University of Toronto, 263 McCaul St, Toronto, ON, Canada. Email: adam.dmytriw@uhn.ca 


\section{INTRODUCTION}

Seizures in the setting of aneurysmal subarachnoid hemorrhage $(\mathrm{SAH})$ are associated with early neurological deterioration and unfavorable functional outcomes. ${ }^{1,2}$ A recent population study estimates that $15 \%$ of SAH patients will have at least one seizure in the first week after their insult, with a cumulative 5-year epilepsy incidence of $12 \% .^{3}$ Multiple potential pathophysiological factors are involved, including increased intracranial pressure, risk for repeat aneurysmal bleeding, neurological deterioration, and increased cerebral metabolic demand. These factors are detrimental to an already injured brain. The use of prophylactic antiepileptic drugs (AEDs) in SAH to prevent such seizure activity is controversial, clinical practices vary widely, ${ }^{4}$ and existing consensus guidelines do not provide clear direciton. ${ }^{5}$

There is paucity of high-quality evidence in the form of wellorganized randomized controlled trials. Notably, seizure rates are comparatively low, the number needed to treat is unknown, and the possible harm associated with AEDs might not be trivial. A recent randomized controlled trial looking at shorter versus extended course AED prophylaxis was halted because of slow recruitment, but did not show a significant difference in seizure development between the treatment groups. Perhaps more worrisome, patients in the extended AED course group had a higher risk for poor functional outcomes, although the small sample size limits the strength of conclusions that can be drawn from this obsevation. ${ }^{6}$ It is thus very important to understand current departmental AED prescribing practices and develop evidence-based protocols for AED prophylaxis in SAH, to improve both patient safety and overall cost-effectiveness.

\section{ObJectives}

We explored clinical and radiological factors associated with the development of seizures and current AED prescribing practices in aneurysmal SAH patients admitted to a high-volume, academic medical-surgical and neuro-intensive care unit (ICU).

\section{Methods}

A retrospective cohort study was thus performed on all patients admitted to a Level 3 ICU (Toronto Western Hospital, Ontario, Canada) with a diagnosis of acute SAH, between January 2012 and January 2014. Institutional review board approval was obtained prior to any data collection, and informed consent was waived due to the retrospective nature of the study. Inclusion criteria were admission to the ICU and a primary diagnosis of acute aneurysmal SAH. Pediatric patients $<18$ years old were excluded as were patients with traumatic SAH.

The following parameters were recorded: clinical characteristics (age, sex, length of hospital, and ICU stay, World Federation of Neurosurgical Societies (WFNS) score at admission, Glasgow Coma Scale (GCS) at presentation, seizure type activity documented at ictus, in-hospital mortality, history of seizures prior to presentation, AED treatment at the time of hospitalization), AED characteristics (prophylactic or therapeutic use, drug class, treatment duration, timing of initiation, discontinuation reason, continuation after discharge), electroencephalography (EEG) status where performed (EEG prior to AED initiation, EEG performed at any point during hospitalization), SAH radiographic characteristics (SAH location, Fisher grade, parenchymal extension, ventricular extension, subdural hematoma, associated contusions, presence of mass effect including hydrocephalus, midline shift (MLS), tonsillar or uncal herniation, aneurysm size and location, primary treatment including clipping, coiling or treatment for arteriovenous malformations (AVMs)).

The primary outcome studied was AED prophylaxis, defined as initiation of AEDs prior to seizure activity. The secondary outcome was clinically confirmed seizure activity during hospitalization. We recorded such witnessed seizure activity as a seizure episode upon chart review if documented clinical observations, muscular activity, and change in conscious state made it plausible. We looked for acute drop in GCS with a postictal phase, tonic-clonic activity, and suspicious motor automatism. Electroencephalographic (EEG) monitoring was performed in patients with consistently low conscious state and no alternate explanation and in those with a high clinical suspicion of seizure activity. Generally initial monitoring was performed over a 30-minute period in the acute setting and repeated if ongoing suspicion of seizure activity persisted despite negative initial EEG. EEG recording was done using the international 10-20 system with 21 scalp electrodes. EEGs were reported by a board certified epileptologist. Focal or generalized epileptiform discharges were recorded as positive for seizure activity.

In accordance with our stated study objectives, detailed descriptive analyses were performed. Across all objectives, continuous variables were summarized with means and standard deviations (SDs) when normally distributed, or with medians and interquartile ranges (IQR) for nonparametric data. Univariate analysis of the data was conducted using two-sample T-tests or Mann-Whitney U-tests as appropriate. Categorical variables were reported as numbers and compared in univariable analysis with the chi-square test. Variables found on univariate analysis as having a $\mathrm{p}$-value of $<0.10$ were incorporated in a multivariate logistic regression model. The model was fitted using forward stepwise automated variable selection with a threshold for inclusion of $\mathrm{p}<0.05$. Post-estimation Pearson goodness-of-fit testing was conducted to evaluate the model. All statistical analyses were performed using Stata 12.0 (Stata Corporation, College Station, TX).

\section{Results \\ Baseline Characteristics}

We identified 68 patients meeting our inclusion criteria. Baseline patient characteristics are presented in Table 1. Mean age $( \pm \mathrm{SD})$ was $62 \pm 12$ years and $42.6 \%$ of patients were male.

Of note, $26.5 \%$ of patients had associated parenchymal hemorrhage, $80.9 \%$ had ventricular extension, and MLS was observed in $17.6 \%$ of patients, with a mean of $9.7 \pm 3.9-\mathrm{mm}$ deviation. There was one patient with a concomitant AVM, along with a ruptured aneurysm. Upon presentation, the median GCS was 10, median WFNS was 4, and median Fisher score was 4 representing a high-grade SAH cohort. Nearly half the patients underwent endovascular intervention and 29 patients (42.6\%) expired before discharge.

\section{Primary Outcome Analysis}

Univariable analyses are presented in Table 2. Twenty-one patients $(30.9 \%)$ received AED prophylaxis during their 
Table 1: Baseline clinical characteristics

\begin{tabular}{|c|c|}
\hline Variables & Patients $(\%)$ \\
\hline Age, years $($ Mean $\pm S D)$ & $62 \pm 12.2$ \\
\hline \multicolumn{2}{|l|}{ Sex } \\
\hline Male & $29(42.6)$ \\
\hline Female & $39(57.4)$ \\
\hline ICU stay $($ Mean $\pm \mathrm{SD})$ & $10.7 \pm 8.9$ \\
\hline Hospital stay (Mean \pm SD) (a) & $29.4 \pm 39.5$ \\
\hline Parenchymal hemorrhage & $18(26.5)$ \\
\hline Ventricular & $55(80.9)$ \\
\hline Subdural & $5(7.4)$ \\
\hline MLS & $12(17.6)$ \\
\hline MLS mm (Mean \pm SD) & $9.7 \pm 3.9$ \\
\hline Hydrocephalus & $43(63.2)$ \\
\hline Herniation & $3(4.4)$ \\
\hline AVM (co-existing) & $1(1.5)$ \\
\hline Aneurysm size (Mean \pm SD) (b) & $6.3 \pm 4$ \\
\hline \multicolumn{2}{|l|}{ Aneurysm location } \\
\hline Acom & $15(24.6)$ \\
\hline ICA & $8(13.1)$ \\
\hline Pcom & $13(21.3)$ \\
\hline MCA & $9(14.8)$ \\
\hline PCA & $2(3.3)$ \\
\hline Posterior circulation & $14(23.0)$ \\
\hline GCS (Median, IQR) (a) & $10(6,14)$ \\
\hline WFNS (Median, IQR) (a) & $4(2,5)$ \\
\hline Fisher (Median, IQR) (a) & $4(4,4)$ \\
\hline \multicolumn{2}{|l|}{ Intervention (a) } \\
\hline None & $17(25.4)$ \\
\hline Surgical & $17(25.4)$ \\
\hline Endovascular & $33(49.3)$ \\
\hline Death & $29(42.6)$ \\
\hline \multicolumn{2}{|l|}{ AED regimen } \\
\hline None & $35(51.5)$ \\
\hline Phenytoin & $23(33.8)$ \\
\hline Phenytoin + Levetiracetam & $10(14.7)$ \\
\hline
\end{tabular}

ICU=intensive care unit; $M L S=$ midline shift; $A V M=$ arteriovenous malformation; GCS=Glasgow Coma Scale; WFNS=World Federation of Neurosurgical Societies; Acom=anterior communicating artery; ICA= internal carotid artery; Pcom=posterior communicating artery; $\mathrm{MCA}=$ middle cerebral artery; $\mathrm{PCA}=$ posterior cerebral artery.

hospitalization. Female gender was associated with AED prophylaxis in univariate analysis with statistical significance $(p$ $=0.036$ ). Multivariate analysis however could not confirm this finding and in fact did not achieve significance (odds ratio (OR) 3.05 with confidence interval (CI) 0.94-9.92 and p-value of 0.064). Pearson goodness-of-fit testing could not be performed because there were not enough degrees of freedom.
Table 2: Factors predicting AED prophylaxis initiation

\begin{tabular}{|c|c|c|c|}
\hline Variables & $\begin{array}{c}\text { AED } \\
\text { prophylaxis } \\
21(30.9 \%)\end{array}$ & $\begin{array}{c}\text { No AED } \\
\text { prophylaxis } \\
47(69.1 \%)\end{array}$ & p-Value \\
\hline Age, years $($ Mean $\pm S D)$ & $65.5 \pm 12.8$ & $60.4 \pm 11.7$ & 0.163 \\
\hline Sex & & & 0.036 \\
\hline Male & $5(17.2)$ & $24(82.8)$ & \\
\hline Female & $16(41)$ & $23(59)$ & \\
\hline ICU stay (Mean \pm SD) & $11.7 \pm 8.8$ & $10.2 \pm 9$ & 0.355 \\
\hline Hospital stay $($ Mean $\pm \mathrm{SD})$ & $31.2 \pm 48.4$ & $28.6 \pm 35.2$ & 0.871 \\
\hline Parenchymal hemorrhage & $3(14.3)$ & $15(31.9)$ & 0.128 \\
\hline Ventricular & $19(90.5)$ & $36(76.6)$ & 0.179 \\
\hline Subdural & $3(14.3)$ & $2(4.3)$ & 0.143 \\
\hline MLS & $1(4.8)$ & $11(23.4)$ & 0.062 \\
\hline MLS mm (Mean \pm SD) & $13 \pm 0$ & $9.4 \pm 4$ & 0.307 \\
\hline Hydrocephalus & $11(52.4)$ & $32(68.1)$ & 0.215 \\
\hline Herniation & $0(0)$ & $3(6.4)$ & 0.236 \\
\hline AVM (co-existing) & $1(4.8)$ & $0(0)$ & 0.132 \\
\hline Aneurysm size $($ Mean $\pm \mathrm{SD})$ & $5.9 \pm 3.1$ & $6.5 \pm 4.3$ & 0.946 \\
\hline Aneurysm location & & & 0.187 \\
\hline Acom & $11(73.3)$ & $4(26.7)$ & \\
\hline ICA & $3(37.5)$ & $5(62.5)$ & \\
\hline Pcom & $8(61.5)$ & $5(38.5)$ & \\
\hline $\mathrm{MCA}$ & $8(88.9)$ & $1(11.1)$ & \\
\hline PCA & $2(100)$ & $0(0)$ & \\
\hline Posterior circulation & $11(78.6)$ & $3(21.4)$ & \\
\hline GCS (median, IQR) & $10(7,15)$ & $10(6,14)$ & 0.450 \\
\hline WFNS (median, IQR) & $4(1,4)$ & $4(2,5)$ & 0.358 \\
\hline Fisher (median, IQR) & $4(4,4)$ & $4(4,4)$ & 0.804 \\
\hline Intervention & & & 0.369 \\
\hline None & $6(28.6)$ & $11(23.9)$ & \\
\hline Surgical & $3(14.3)$ & $14(30.4)$ & \\
\hline Endovascular & $12(57.1)$ & $21(45.7)$ & \\
\hline Death & $12(57.1)$ & $17(36.2)$ & 0.106 \\
\hline
\end{tabular}

ICU=intensive care unit; MLS=midline shift; AVM=arteriovenous malformation; GCS=Glasgow Coma Scale; WFNS=World Federation of Neurosurgical Societies; Acom=anterior communicating artery; ICA=internal carotid artery; Pcom=posterior communicating artery; $\mathrm{MCA}=$ middle cerebral artery; PCA=posterior cerebral artery.

Patients with MLS seemed less likely to receive AED prophylaxis initiation when compared to patients without MLS (p-value of 0.062 on univariate analysis). This remained nonsignificant on multivariate analysis (OR 0.19 with CI 0.02-1.6 and p-value of 0.126 ) when correcting for patient sex.

Factors tested but not showing statistical significance were age, length of hospital or ICU stay, presence of parenchymal or ventricular extension, subdural hemorrhage, millimeters of MLS, hydrocephalus, brain herniation, co-existence of AVM, aneurysm size, GCS, Fisher grade, type of intervention, and mortality. 


\section{Secondary Outcome Analysis}

Univariate analysis regarding the secondary outcome of experiencing clinical and/or electrographic seizures at any point during hospitalization is presented in Table 3. Eighteen patients (26.5\%) experienced seizures during their hospitalization as reported by medical staff, bystanders, or first responders. Factors showing or approaching statistical significance in univariate analysis were the existence of subdural hematoma $(\mathrm{p}=0.077)$, the presence of MLS $(p=0.006)$, and a higher WFNS score $(p=0.081)$. Further examination through multivariate analysis, however, found only MLS to independently predict clinical seizures during hospitalization (OR, 5.09; CI, 1.07-24.08; p-value, 0.04), incorporating/controlling for the above factors. Post-estimation goodness of fit revealed satisfactory model fitting $(\mathrm{p}=0.97)$.

In our patient cohort, we identified 18 patients that had a witnessed clinical seizure during their admission. Of those, four patients had a seizure-like episode prior to their arrival at the Emergency Department (ED), as witnessed by a bystander or emergency medical services (EMS). Two patients had seizures witnessed in the ED and 12 patients experienced seizures during their ICU in-hospital stay. Notably, of the 12 patients who had at least one seizure in the ICU, 4 patients had no clinical evidence of seizure activity and were diagnosed on EEG alone.

\section{AED Treatment Duration}

Of the 33 patients where AED prophylaxis or treatment was initiated, 25 patients (75.8\%) had their AED discontinued prior to discharge and 8 patients (24.2\%) were discharged on an AED. Of those, only three were still taking an AED at first follow-up. In all, 15 patients received AED on the day of arrival, 10 the next day, 2 the second day, and 1 each after 3, 4, 6, 810 , and 14 days.

The average duration to starting AED prophylaxis was 1.79 days (SD 3.23) and prophylaxis was continued for a median of 9 days (IQR 4-21) during the inpatient stay.

\section{Discussion}

Seizures in the setting of aneurysmal SAH have been the subject of much debate. Lin et al. previously found that seizures at SAH onset predicted significant neurologic deficits. ${ }^{7}$ Similarly, Claassen et al. found that seizures following SAH may lead to higher mortality rates and disability. ${ }^{8}$ Conversely, other groups have concluded that early seizures after SAH do not have a significant impact on outcome, but are rather a marker of severity of the underlying brain injury. ${ }^{9}$ In this study, we evaluated factors associated with prescribing AED prophylaxis in SAH patients and risk factors for actual seizures during hospitalization, to see if the two overlap.

\section{Patterns of AED Use}

Multivariate analysis in our study identified only female gender to be independently associated with higher AED prescription rates. However, this finding was not significant $(p=0.06)$ and the confidence interval for the odds ratio crossed 1 . We also ran a stepwise model selection for all variables with univariate $\mathrm{p}$-values of $<0.20$. This revealed statistical significance for female patients and patients who died, being more likely to have

\section{Table 3: Factors predicting seizures}

\begin{tabular}{|c|c|c|c|}
\hline Variables & $\begin{array}{c}\text { Seizure } \\
18(26.5 \%)\end{array}$ & $\begin{array}{l}\text { No Seizure } \\
50(73.5 \%)\end{array}$ & p-Value \\
\hline Age, years $($ Mean $\pm S D)$ & $62.2 \pm 13.3$ & $61.9 \pm 11.9$ & 0.835 \\
\hline Sex & & & 0.707 \\
\hline Male & $7(24.1)$ & $22(75.9)$ & \\
\hline Female & $11(28.2)$ & $28(71.8)$ & \\
\hline ICU stay $($ Mean $\pm \mathrm{SD})$ & $14.7 \pm 11.4$ & $9.2 \pm 7.4$ & 0.129 \\
\hline Hospital stay (Mean \pm SD) & $38.6 \pm 49.6$ & $26.1 \pm 35$ & 0.497 \\
\hline Parenchymal hemorrhage & $6(33.3)$ & $12(24)$ & 0.442 \\
\hline Ventricular & $16(88.9)$ & $39(78)$ & 0.314 \\
\hline Subdural & $3(16.7)$ & $2(4)$ & 0.077 \\
\hline MLS & $7(38.9)$ & $5(10)$ & 0.006 \\
\hline MLS mm (Mean \pm SD) & $10.3 \pm 3.2$ & $8.8 \pm 5.1$ & 0.567 \\
\hline Hydrocephalus & $13(72.2)$ & $30(60)$ & 0.356 \\
\hline Herniation & $2(11.1)$ & $1(2)$ & 0.107 \\
\hline AVM (co-existing) & $0(0)$ & $1(2)$ & 0.546 \\
\hline Aneurysm size $($ Mean $\pm \mathrm{SD})$ & $6.2 \pm 3.7$ & $6.3 \pm 4.1$ & 0.891 \\
\hline Aneurysm location & & & 0.421 \\
\hline Acom & $9(60.0)$ & $6(40.0)$ & \\
\hline ICA & $8(100)$ & $0(0)$ & \\
\hline Pcom & $10(76.9)$ & $3(23.1)$ & \\
\hline $\mathrm{MCA}$ & $6(66.7)$ & $3(33.3)$ & \\
\hline PCA & $1(50.0)$ & $1(50.0)$ & \\
\hline Posterior circulation & $10(71.4)$ & $4(28.6)$ & \\
\hline GCS (Median, IQR) & $8(5,11)$ & $10(6,15)$ & 0.187 \\
\hline WFNS (Median, IQR) & $4(4,5)$ & $4(1,5)$ & 0.081 \\
\hline Fisher (median) & $4(4,4)$ & $4(4,4)$ & 0.563 \\
\hline Intervention & & & 0.610 \\
\hline None & $3(16.7)$ & $14(28.6)$ & \\
\hline Surgical & $5(27.8)$ & $12(24.5)$ & \\
\hline Endovascular & $10(55.6)$ & $23(46.9)$ & \\
\hline Death & $8(44.4)$ & $21(42)$ & 0.857 \\
\hline
\end{tabular}

ICU=intensive care unit; MLS=midline shift; AVM=arteriovenous malformation; GCS=Glasgow Coma Scale; WFNS=World Federation of Neurosurgical Societies; Acom=anterior communicating artery; ICA= internal carotid artery; Pcom=posterior communicating artery; $\mathrm{MCA}=$ middle cerebral artery; $\mathrm{PCA}=$ posterior cerebral artery.

received AED therapy. Overall, it would seem plausible that this is a chance finding due to type II error and unequal baseline distribution (female $57.4 \%$ vs. male $42.6 \%$ ) rather than an active decision-making process at presentation, since female gender is not a known risk factor to develop seizures in SAH and there is no official recommendation regarding AED initiation and gender.

The use of prophylactic AEDs in SAH to prevent seizure activity is controversial and clinical practices currently vary widely. ${ }^{4}$ In a recent survey distributed to 25 high-volume SAH centers, seizure prophylaxis was endorsed by half, while $42 \%$ opposed it. ${ }^{10}$ This observation is consistent with prior 

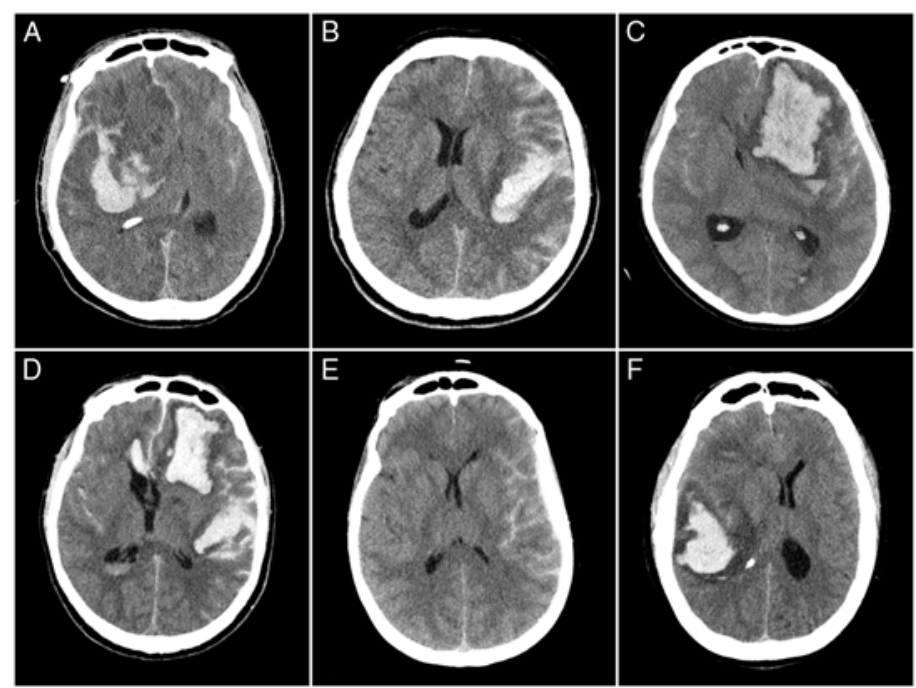

Figure 1: Unenhanced $C T$ of the head demonstrating $(A-B)$ diffuse, $(C)$ left basal ganglia, (D) left temporal, $(E)$ left Sylvian, and $(F)$ right temporal hemorrhage patterns in our Level 1 cohort. All of these patients exhibited $M L S$ and were subsequently found to have seizure activity.

observational data reporting AED use for $65 \%$ of SAH patients. ${ }^{4}$ That same group noted that the prescribing pattern seemed arbitrary and mostly physician-dependent. Prescribers were more likely to prescribe AED in patients of younger age, with worse neurological grades or lower systolic blood pressure on admission. These factors were not associated with AED prescribing in our cohort. That said, we did not look at admission blood pressure. Adding to the confusion and wide variability in clinical practice, the $2012 \mathrm{SAH}$ consensus guidelines are somewhat vague regarding AED use. ${ }^{5}$ The guidelines suggest that the use of prophylactic anticonvulsants may be considered in the immediate post-hemorrhagic period. However, routine long-term use of anticonvulsants is not recommended, apart from certain at-risk groups.

Our study associated female gender with higher AED prophylaxis prescribing practices. This is not a known at-risk group, and no official recommendation exists to support preferential AED prescribing for the female gender. Patients with MLS on their initial computer tomography (CT; Figure 1) seemed less likely to receive AED prophylaxis compared to those without. Whilst there is no official recommendation commenting on AED prophylaxis in SAH patients with MLS specifically, it would certainly seem counterintuitive to preferentially prescribe such drug therapy to the cohort without MLS. As discussed above with gender preference, it certainly becomes very difficult to explain this finding.

\section{Discrepancy between Seizure Predictors and AED Prophylaxis}

An exploratory analysis of CONSCIOUS-1 did find an association between MLS and the occurrence of seizures on univariate but not multivariate analysis. ${ }^{11}$ Seizures have also been reported to independently worsen MLS after a hemorrhagic stroke, ${ }^{12}$ which is conceptually in line with our findings.

Multivariate analysis in our SAH cohort identified MLS to be the only independent predictor of seizures during hospitalization.
While existence of a subdural hematoma and worse WFNS grade were also predictive of seizures in univariate analysis, only MLS was significant in multivariate. This remained true in an adjusted multivariate analysis for univariate $\mathrm{p}$-values of $<0.20$ with the exception of ICU length of stay also becoming significantly associated with the chance to develop seizures.

As discussed earlier, MLS was not significantly associated with AED prophylaxis on multivariate analysis. This discrepancy between factors predictive of seizures and factors associated with the actual clinical practice of AED prophylaxis prescription is emblematic of the controversy that exists regarding AED prescribing in SAH. Due to the small sample size and the retrospective nature of our cohort, larger and more robust studies are recommended to definitively investigate the risk factors for seizures in SAH, to aid clinicians in making informed decisions about AED prophylaxis.

\section{Adverse Effects of Prophylactic AED}

In our cohort, none of the patients on an AED had their drug discontinued due to adverse effects. In two cases, AED prophylaxis was suspended due to supratherapeutic levels without neurological symptoms however.

A single-center retrospective study in 2000 found that $99 \%$ of SAH patients were started on AED during their hospital stay. The duration of therapy ranged from 1 to 68 days. Seizures occurred in four patients (4.1\%), three of whom were on AEDs with levels in the therapeutic range at the time of seizure. ${ }^{13}$ Such AED prophylaxis was associated with an increased risk of in-hospital complications and worse outcome as measured by the Glasgow Outcome Score (GOS). Similarly, Naidech et al. found an association between phenytoin exposure after SAH and functional as well as cognitive disability. ${ }^{14}$ Phenytoin has also been linked to drug-induced fever and vasospasm and its interaction with nimodipine is well-studied. ${ }^{15}$ Similar detrimental effects have been found in volunteer and animal studies. A volunteer study conducted nearly 20 years ago showed that AEDs were able to 
impair cognitive function in healthy volunteers. ${ }^{16}$ The use of AEDs in animal models of induced brain injury has been shown to cause neurodegeneration and impaired recovery. ${ }^{17}$ The mechanisms underlying possible harm from AEDs are poorly understood but may be secondary to reduced neuronal irritability, which could lead to diminished synaptic growth and, therefore, reduced potential for neurological recovery. ${ }^{18}$

Whilst it would seem that AED prophylaxis was well tolerated in our cohort, it is important to note that our study is of a retrospective nature and as such adverse affects were not recorded prospectively and might well have been underestimated.

\section{Comparison of AED}

In the present study, 23 patients were prescribed phenytoin and 10 received combination phenytoin and levetiracetam.

Antiepileptic therapy with phenytoin is fraught with adverse effects, including deranged liver function, thrombocytopenia, rash, and Stevens-Johnson syndrome. ${ }^{19}$ Furthermore, the wide range of neuropsychological side effects shown to be associated with phenytoin, as well as the known interaction with various drugs on a pharmacokinetic level including nimodipine, have led several centers to move away from phenytoin and toward newer agents such as levetiracetam. The interaction of phenytoin with nimodipine seems particularly concerning in SAH, where delayed cerebral ischemia can impart devastating consequences and the single prophylactic measure shown to be of benefit is the very same drug. Evidence to support agents such as levetiracetam is also lacking. However, several reports and uncontrolled studies report good tolerability and potentially favorable long-term neurological outcomes. ${ }^{20,21}$

\section{Duration of AED Therapy}

Another concern with non-protocolized AED prescription practices is the speed of drug discontinuation, when no longer necessary, to prevent unnecessary drug interactions, complications, and cost. We looked at the duration of AED prescription in our cohort and found that only eight patients were discharged with an AED, most of which were stopped at the first follow-up appointment. It seems that, while AED prescribing might indeed be arbitrary, medical professionals and pharmacists are likely aware of possible associated problems and seem proactive in stopping AED therapy when no longer required.

\section{Study Limitations}

Our study is retrospective and based on chart and imaging reviews, which brings about the usual problems and limitations associated with retrospective work. Numbers would generally be considered to be limited, and we have been examining a patient cohort with very heterogenous clinical courses and radiological patterns. This certainly makes it difficult to draw strong conclusions. Nevertheless, it is important to note that this ultimately represents a sick high-grade SAH cohort based on the WFNS, Fisher, and mortality data and as such makes it very relevant for the question asked. Another limitation was that none of the investigators collecting the data were blinded to any aspects of the data (i.e. clinical, radiological, seizure development, or AED status), which could have introduced a bias. Similarly, the epileptologists were of course not blinded to the
AED status at the time of the EEG, since they were asked to report on the EEG based on a clinical question. With regards to the EEG recording, it is current standard of practice in our center to only order an EEG, if there is a suspicion of seizures or protracted low conscious level without an alternate explanation. Therefore, it is certainly plausible that some of the patients might have had evidence of radiographic seizures, if all the patients had received at least one EEG. This could represent a limitation but on the other hand represents more closely current practice in many centers around the world, where EEG and continuous EEG are a scarce and not readily available resource. AED dosages were also not recorded.

\section{Current Practices and Future Direction}

Whether SAH patients require prophylactic AED treatment and the optimal regimen thereof currently remain unanswered. Moreover, there is paucity of high-quality evidence, in the form of well-organized randomized controlled trials. Seizures in the setting of acute brain injury and unsecured aneurysms certainly have detrimental effects on the brain, regardless of the causeeffect question. Such unnecessary secondary injury should be avoided, if possible. However, seizure rates are comparatively low, the number needed to treat is unknown and the possible harm associated with AEDs as outlined above might not be trivial. Studies such as the systematic review by Raper et al., ${ }^{19}$ which was unable to show a difference in post-SAH seizure incidence between the AED and non-AED cohorts, add to the growing evidence that we might not be doing patients a favor by indiscriminately initiating AED prophylaxis. To date, most studies that have examined the prevalence of AED prophylaxis in SAH lack information on the regimen and intensity of treatment and when best to terminate prophylaxis. Therefore, it is of paramount importance to understand current departmental AED prescribing practices, in order to develop evidence-based protocols for AED prophylaxis in SAH, to improve both patient safety and overall cost-effectiveness. In this study, we show the discrepancy between factors predicting AED prophylaxis practices and actual risk factors to predict seizure activity during hospitalization. We hope that future studies will better delineate the risk factors involved, for clinicians to make informed choices regarding AED in SAH. Specifically, we believe that future studies should look at subarachnoid clot burden or Fisher scale, subdural hematomas, and MLS and their association with seizure occurrence. On a national level, a first step could include a similar expanded retrospective study including other Canadian SAH centers, in order to produce a national practice audit. Additional and informative data could be gathered through a Canadian wide survey of current AED prophylaxis practice in aneurysmal SAH targeting neurology, neurosurgery, and the critical care communities.

With most large-volume subarachnoid centers securing their aneurysms in the first 24 hours, the clinical situation of seizure prophylaxis in the unsecured aneurysm has become less frequent and relevant. As such a prospective randomized trial examining the value of AED in the secured SAH patient cohort should really become a reality in the near future, with the controversy continuing and a lot of centers likely to still have equipoise on the issue. 


\section{Conclusion}

Our study suggests an association of MLS with seizure activity; however AED prophylaxis was not informed by this risk factor. Conversely, female SAH patients were more likely to receive AEDs, but no increased risk was detected in this group. This result underscores a discrepancy in AED prescribing practices in SAH. Larger studies are needed to identify the risk factors for seizures in the setting of SAH and assist in informed decision-making.

\section{Statement of Authorship}

AAD designed and conceptualized study; analyzed and interpreted data; and drafted manuscript for intellectual content. GAM analyzed and interpreted data, and revised manuscript for intellectual content. JZ revised manuscript for intellectual content. JMS revised manuscript for intellectual content. MEW designed and conceptualized study; analyzed data; and revised manuscript for intellectual content. SS designed and conceptualized study; analyzed data; and revised manuscript for intellectual content.

\section{Disclosures}

The authors have no conflicts of interest to declare.

\section{REFERENCES}

1. Butzkueven H, Evans AH, Pitman A, et al. Onset seizures independently predict poor outcome after subarachnoid hemorrhage. Neurology. 2000;00(0):1315-20.

2. Huff JS, Perron AD. Onset seizures independently predict poor outcome after subarachnoid hemorrhage. Neurology. 2001;56(10):1423-4.

3. Huttunen JM, Kurki MI, von und zu Fraunberg M, et al. Epilepsy after aneurysmal subarachnoid hemorrhage: a population-based, long-term follow-up study. Neurology. 2015;85(22):1997.

4. Rosengart AJ, Huo JD, Tolentino J, et al. Outcome in patients with subarachnoid hemorrhage treated with antiepileptic drugs. J Neurosurg. 2007;107(2):253-60.

5. Connolly ES, Jr., Rabinstein AA, Carhuapoma JR, et al. Guidelines for the management of aneurysmal subarachnoid hemorrhage: a guideline for healthcare professionals from the American Heart
Association/American Stroke Association. Stroke. 2012;43(6): 1711-37.

6. Human T, Diringer MN, Allen M, et al. A randomized trial of brief versus extended seizure prophylaxis after aneurysmal subarachnoid hemorrhage. Neurocrit Care. 2018;28(2):169-74.

7. Lin CL, Dumont AS, Lieu AS, et al. Characterization of perioperative seizures and epilepsy following aneurysmal subarachnoid hemorrhage. J Neurosurg. 2003;99(6):978-85.

8. Claassen J, Perotte A, Albers D, et al. Nonconvulsive seizures after subarachnoid hemorrhage: multimodal detection and outcomes. Ann Neurol. 2013;74(1):53-64.

9. Pinto AN, Canhao P, Ferro JM. Seizures at the onset of subarachnoid haemorrhage. J Neurol. 1996;243(2):161-4.

10. Dewan MC, Mocco J. Current practice regarding seizure prophylaxis in aneurysmal subarachnoid hemorrhage across academic centers. J Neurointerv Surg. 2015;7(2):146-9.

11. Vespa PM, O'Phelan K, Shah M, et al. Acute seizures after intracerebral hemorrhage: a factor in progressive midline shift and outcome. Neurology. 2003;60(9):1441-6.

12. Ibrahim GM, Fallah A, Macdonald RL. Clinical, laboratory, and radiographic predictors of the occurrence of seizures following aneurysmal subarachnoid hemorrhage. J Neurosurg. 2013;119(2): $347-52$.

13. Rhoney DH, Tipps LB, Murry KR, Basham MC, Michael DB, Coplin WM. Anticonvulsant prophylaxis and timing of seizures after aneurysmal subarachnoid hemorrhage. Neurology. 2000; 55(2):258-65.

14. Naidech AM, Kreiter KT, Janjua N, et al. Phenytoin exposure is associated with functional and cognitive disability after subarachnoid hemorrhage. Stroke. 2005;36(3):583-7.

15. Bleck TP, Chang CW. Ten things we hate about subarachnoid hemorrhage (or, the taming of the aneurysm). Crit Care Med. 2006;34(2):571-4.

16. Behavioral and cognitive effects of anticonvulsant therapy. American academy of pediatrics committee on drugs. Pediatrics. 1995;96(3 Pt 1):538-40.

17. Macdonald RL, Kelly KM. Antiepileptic drug mechanisms of action. Epilepsia. 1995;36 (Suppl 2):S2-12.

18. Meador KJ. Neurodevelopmental effects of antiepileptic drugs. Curr Neurol Neurosci Rep. 2002;2(4):373-8.

19. Raper DM, Starke RM, Komotar RJ, Allan R, Connolly ES, Jr. Seizures after aneurysmal subarachnoid hemorrhage: a systematic review of outcomes. World Neurosurg. 2013;79(5-6):682-90.

20. Shah D, Husain AM. Utility of levetiracetam in patients with subarachnoid hemorrhage. Seizure. 2009;18(10):676-9.

21. Szaflarski JP, Sangha KS, Lindsell CJ, Shutter LA. Prospective, randomized, single-blinded comparative trial of intravenous levetiracetam versus phenytoin for seizure prophylaxis. Neurocrit Care. 2010;12(2):165-72. 